\title{
Numerical Investigation of Phenol Extraction using Liquid-Liquid Stratified Flow in a T shaped Micro-Channel
}

\author{
Rahul Antony \\ Dept. of Chemical Engg. \\ NIT Calicut, Kerala \\ Arun $\mathrm{G}$ \\ Dept. of Chemical Engg. \\ IISc, Bangalore
}

\author{
Anu N \\ Dept. of Chemical Engg. \\ NIT Calicut, Kerala \\ N Selvaraju \\ Dept. of Chemical Engg. \\ NIT Calicut, Kerala
}

\begin{abstract}
Numerical investigation of the extraction process in a $\mathrm{T}$ shaped micro channel makes the model for the extraction proces, for increased extraction efficiency an enhanced mass transfer between the two phases. The extraction rate can be controlled precisely by adjusting operational parameters such as the flow rate and the flow rate ratio. Here the work studied the extraction of phenol from dodecane using water as solvent. Process is modelled and the extraction ratios are compared with the experimental data published by Okubo et al [14]. Different modelling approaches attempted include pseudo mass transfer coefficient model (1D model) and two dimensional unsteady state diffusion model. IN pseudo mass transfer coefficient model a suitable value of mass transfer coefficient is chosen so as to match experimental results. Hence the model provides a method for predicting mass transfer coefficient. Two dimensional unsteady state diffusion models is comprehensive and considers diffusion in both directions. The diffusivities are calculated using the Wile Chang equation. Predictions of all the three models at different velocities are tabulated and compared.
\end{abstract}

\section{General Terms}

Microfluidics, Stratified Flow Model

\section{Keywords}

Microchannel Extraction process, Model for T shaped microchannel extraction system, Matlab based modelling for Stratified Flow in a Microchannel.

\section{INTRODUCTION}

Microfluidics: As the name indicates this is a new area which is related to fluid properties inside a micro channel. It is the science and technology of systems that process or manipulate small (10-9 to 10-18 litres) amounts of fluids, using channels with dimensions of tens to hundreds of micro meters ${ }^{[7]}$. Now a day this area is very hot for researchers because which has the potential to influence subject areas from chemical synthesis and biological analysis to optics and information technology. Recently the area Microfluidics is related to Chemical Engineering, Mechanical Engineering Branches. As per the words of Anand ${ }^{[1]}$, "Microfluidics is the manipulation and control of fluids in small scale, and has heralded a new age in science as evidenced by the rapid increase in the amount and quality of academic and industrial research output in this area in the recent times."

The property of a fluid flow through a micro channel is very different with the normal flow. The applications of this area are too vast and some of the applications are belongs to cartelistic reactors ${ }^{[2,3]}$, Tissue Engineering ${ }^{[4]}$, Multiphase mixing and reaction ${ }^{[5]}$, Blood Flow and the drug reaction ${ }^{[6]}$, highthroughput screening in drug development ${ }^{[9,10]}$, bio-analyses [11], examination and manipulation of samples consisting of a single cell ${ }^{[12,13]}$, and also in efficient heat sinks ${ }^{[8]}$. But one of the main applications of this field is the extraction process. The enhanced mass transfer and the increased extraction efficiency of the micro channel extraction process calls for the attention of scientists. 
For extracting product materials to another phase, the parallel reactions can be used. The liquid - liquid extraction depends on the mass transfer flux, residence time and the area of liquid liquid interface. So the slug regime is the best option for the extraction process and stratified flow also can be the best for the extraction process. The main aim of this study is to identify the advantage and disadvantage of liquid-liquid extraction using $\mathrm{T}$ channel stratified flow fluid operations using micro-reactors.

\section{EXPERIMENTAL}

Liquid-liquid extraction is a method to separate compounds based on their relative solubility's in two different immiscible liquids. There are two steps in the extraction process. One is extracting useful substances from one phase to another and the second is separating the mixture into two phases

Here we are considering, Okubo ${ }^{[14]}$ et al's work, the reaction between Water and Dodecane in a T micro-channel:

The Y-shaped micro channel used is fabricated from glass. The micro channel was 400 micro m wide, 90 micro m deep and 33mmlong. Dodecane (Wako Pure Chemical Industries) with concentration 1000ppm phenol (Wako Pure Chemical Industries) was prepared. Extraction experiments were performed by feeding distilled water and 1000ppm phenol/ dodecane into the micro channel at the same flow rate. The concentration of phenol in water phase, $\mathrm{Cw}$ was measured by using UV- vis spectrometer (Multispec 1500, Shimadzu). Phenol has its typical peek at $270 \mathrm{~nm}$ in water. The concentration of phenol was determined by the calibration curve method. Under the condition of above mentioned, the total flow rate was changed. The retention time was approximately from 0.1 to 1 s. $^{[14]}$

\section{MATHEMATICAL MODEL FOR EXTRACTION OF PHENOL}

Convection Diffusion Equation: As far as our case (extraction) is considered we have diffusion in both the directions but convection only in the flow direction. In the case of extraction the equation is simplified by the fact that there is no reaction taking place. Therefore the unsteady state convection diffusion equation reduces to

$$
\begin{array}{r}
\mathbf{D}_{w} \frac{\partial^{2} \mathbf{C}_{w}}{\partial \mathbf{x}^{2}}+\mathbf{D}_{w} \frac{\partial^{2} \mathbf{C}_{w}}{\partial \mathbf{y}^{2}}-\mathbf{U}_{w} \frac{\partial \mathbf{C}_{w}}{\partial \mathbf{x}}=\frac{\partial \mathbf{C}_{w}}{\partial t} \\
\mathbf{D}_{d} \frac{\partial^{2} \mathbf{C}_{d}}{\partial \mathbf{x}^{2}}+\mathbf{D}_{d} \frac{\partial^{2} \mathbf{C}_{d}}{\partial \mathbf{y}^{2}}-\mathbf{U}_{d} \frac{\partial \mathbf{C}_{d}}{\partial \mathbf{x}}=\frac{\partial \mathbf{C}_{d}}{\partial t}
\end{array}
$$

Cross stream boundary conditions:

$\mathbf{a t x}=\mathbf{x} \mathrm{s}$

$\mathbf{C}_{\mathrm{w}}=\mathbf{P C}_{\mathrm{d}}$

$\mathbf{D}_{\mathbf{w}} \frac{\partial \mathbf{C}_{w}}{\partial \mathbf{y}}=\mathbf{D}_{\mathbf{d}} \frac{\partial \mathbf{C}_{\mathbf{d}}}{\partial \mathbf{y}}$

Streamwise boundary conditions:-(Danckwertz boundary conditions)

$$
\left(\frac{\partial C}{\partial x}\right)_{x=L}=0
$$

$$
\left(\mathbf{u C}_{\mathbf{i}}=\mathbf{u C}-\mathbf{D}_{\mathrm{AB}}\left(\frac{\partial \mathrm{C}}{\partial \mathrm{x}}\right)\right)_{\mathrm{x}=0}
$$

Further the transverse diffusion term (second term) may be replaced by a pseudo mass transfer coefficient which accounts for net mass transfer between the phases .Resulting in a 1D models given below:

$$
\begin{aligned}
& \mathbf{D}_{\mathbf{w}} \frac{\partial^{2} \mathbf{C}_{w}}{\partial \mathbf{x}^{2}}-\mathbf{U}_{\mathrm{w}} \frac{\partial \mathbf{C}_{\mathbf{w}}}{\partial \mathbf{x}}+\mathbf{K}_{\mathbf{m}}\left(\mathbf{P} \mathbf{C}_{\mathbf{d}}-\mathbf{C}_{\mathrm{w}}\right)=0 \\
& \mathbf{D}_{\mathrm{d}} \frac{\partial^{2} \mathbf{C}_{\mathrm{d}}}{\partial \mathbf{x}^{2}}-\mathbf{U}_{\mathrm{d}} \frac{\partial \mathbf{C}_{\mathrm{d}}}{\partial \mathbf{x}}-\mathbf{K}_{\mathbf{m}}\left(\mathbf{C}_{\mathbf{d}}-\mathbf{C}_{\mathrm{w}} / \mathbf{P}\right)=0 \\
& \text { Where, } \\
& \mathrm{PC}_{\mathrm{d}}=\mathrm{C}_{\mathrm{w}} \text { is the equlibrium relation }
\end{aligned}
$$

\subsection{Extraction Ratio}

Extraction ratio is an important measure of the efficiency of the extraction process.

\section{Extraction ratio $=$ \\ Concentration of solute transfered to solvent form carrier Concnetration of solute initially present in carrier \\ Extraction ratio is an important parameter for} comparing the experimental and predicted data in the present study it is calculated at a time when the feed given at the inlet reaches the outlet. It is clear from the convection diffusion equation that the convection diffusion equation depends upon the velocity across the cross section. Hence we have to solve for 
the velocity profile (momentum equation) then substitute that velocity in the convection diffusion equation.

\subsection{Adjacent flow of two immiscible}

\section{fluids and its model}

Consider two immiscible, incompressible fluids flowing in $\mathrm{z}$ direction in a horizontal thin slit of length $\mathrm{L}$ and width $\mathrm{W}$ under the influence of a horizontal pressure gradient.

The fluid flow rates are adjusted so that the slit is half filled with fluid1 (the more dense phase) and half filled with fluid2 (the less dense phase). ${ }^{[15]}$

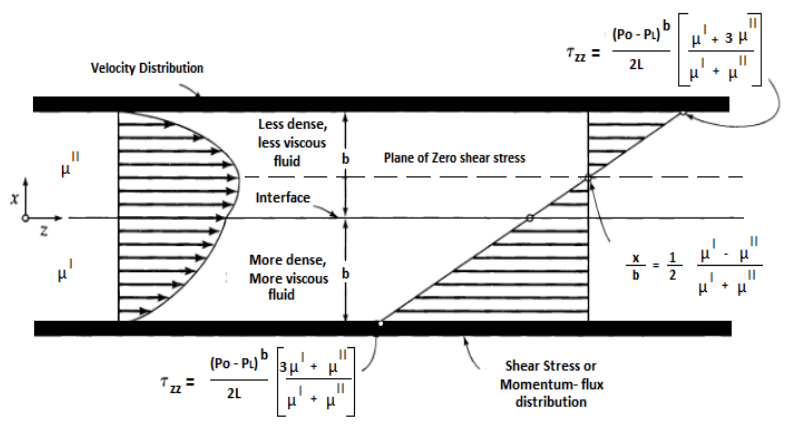

Fig 1: Adjacent flow of two immiscible fluids ${ }^{[15]}$

A differential momentum balance leads to the following differential equation: $\frac{\mathbf{d} \tau_{\mathbf{x z}}}{\mathbf{d x}}=\frac{\mathbf{p}_{0}-\mathbf{p}_{\mathbf{L}}}{\mathbf{L}}$

This equation is obtained for both phase1 and phase2. On integration, the equation becomes:

$$
\begin{aligned}
\tau_{\mathrm{xz}}^{1} & =\left(\frac{\mathbf{p}_{0}-\mathbf{p}_{\mathrm{L}}}{\mathbf{L}}\right) \mathbf{x}+\mathbf{C}_{1}^{1} \\
\tau_{\mathrm{xz}}^{\square} & =\left(\frac{\mathbf{p}_{0}-\mathbf{p}_{\mathrm{L}}}{\mathbf{L}}\right) \mathrm{x}+\mathbf{C}_{1}^{\square}
\end{aligned}
$$

\section{Boundary conditions}

$$
\begin{aligned}
& \text { At } \mathbf{x}=\mathbf{0} ; \boldsymbol{\tau}_{\mathbf{x z}}^{\mathbf{1}}=\boldsymbol{\tau}_{\mathbf{x z}} \\
& \text { Therefore } \quad \boldsymbol{C}_{1}^{1}=\boldsymbol{C}_{1}
\end{aligned}
$$

Substituting Newton's law of viscosity, we get

$$
\begin{aligned}
& -\mu^{1} \frac{d v_{\mathbf{z}}^{1}}{\mathbf{d x}}=\left(\frac{\mathbf{p}_{0}-\mathbf{p}_{\mathbf{L}}}{\mathbf{L}}\right) \mathbf{x}+\mathbf{C}_{1} \\
& -\mu^{\square} \frac{\mathbf{d v _ { \mathbf { z } }}}{\mathbf{d x}}=\left(\frac{\mathbf{p}_{0}-\mathbf{p}_{\mathbf{L}}}{\mathbf{L}}\right) \mathbf{x}+\mathbf{C}_{1}
\end{aligned}
$$

On integrating, we get

$$
\begin{aligned}
& \mathbf{v}_{\mathbf{z}}^{1}=-\left(\frac{\mathbf{p}_{0}-\mathbf{p}_{\mathbf{L}}}{2 \boldsymbol{\mu} \mathbf{L}}\right) \mathbf{x}^{2}-\frac{\mathbf{C}_{1}}{\boldsymbol{\mu}^{1}} \mathbf{x}+\mathbf{C}_{2}^{1} \\
& \mathbf{v}_{\mathbf{z}}^{\square}=-\left(\frac{\mathbf{p}_{0}-\mathbf{p}_{\mathbf{L}}}{2 \boldsymbol{\mu} \mathbf{L}}\right) \mathbf{x}^{2}-\frac{\mathbf{C}_{1}}{\boldsymbol{\mu}^{\square}} \mathbf{x}+\mathbf{C}_{2}^{\square}
\end{aligned}
$$

The 3 integration constants can be determined from the following boundary conditions:
1. At $\mathrm{x}=\mathbf{0} ; \quad \boldsymbol{v}_{z}^{1}=\boldsymbol{v}_{z}^{\square}$
2. At $\mathrm{x}=-\mathrm{b} ; \quad v_{z}^{1}=0$
3. At $x=+b ; \quad v_{z}^{\square}=0$

On applying these boundary conditions, we get

1. $\mathrm{C}_{2}^{1}=\mathrm{C}_{2}^{\square}$

2. $0=-\left(\frac{p_{0}-p_{L}}{2 \mu^{1} L}\right) b^{2}+\frac{C_{1}}{\mu^{1}} b+C_{2}^{1}$

3. $0=-\left(\frac{\mathrm{p}_{0}-\mathrm{p}_{\mathrm{L}}}{2 \mu \mathrm{L}}\right) \mathrm{b}^{2}-\frac{\mathrm{C}_{1}}{\mu^{\square}} \mathrm{b}+\mathrm{C}_{2}^{\square}$

Therefore,

$$
\begin{aligned}
& C_{1}=-\left(\frac{p_{0}-p_{L}}{2 L}\right) b\left(\frac{\mu^{1}-\mu^{\square}}{\mu^{1}+\mu^{\square}}\right) \\
& C_{2}=-\left(\frac{p_{0}-p_{L}}{2 \mu^{1} L}\right) b^{2}\left(\frac{2 \mu^{1}}{\mu^{1}+\mu^{\square}}\right)=C_{2}^{\square}
\end{aligned}
$$

The resulting velocity profile is given by ${ }^{[15]}$ :

$$
\begin{aligned}
& \mathbf{v}_{\mathbf{x z}}^{1}=\left(\frac{\mathbf{p}_{0}-\mathbf{p}_{\mathbf{L}}}{2 \boldsymbol{\mu}^{1} \mathbf{L}}\right) \mathbf{b}^{2}\left[\left(\frac{2 \boldsymbol{\mu}^{1}}{\boldsymbol{\mu}^{1}+\boldsymbol{\mu}^{\square}}\right) \mathbf{x}+\left(\frac{\boldsymbol{\mu}^{1}-\boldsymbol{\mu}}{\boldsymbol{\mu}^{1}+\boldsymbol{\mu}}\right) \frac{\mathbf{x}}{\mathbf{b}}-\left(\frac{\mathbf{x}}{\mathbf{b}}\right)^{2}\right] \\
& \mathbf{v}_{\mathbf{z}}^{\square}=\left(\frac{\mathbf{p}_{0}-\mathbf{p}_{\mathbf{L}}}{2 \boldsymbol{\mu} \mathbf{L}}\right) \mathbf{b}^{2}\left[\left(\frac{2 \boldsymbol{\mu}}{\boldsymbol{\mu}^{1}+\boldsymbol{\mu}}\right) \mathbf{x}+\left(\frac{\boldsymbol{\mu}^{1}-\boldsymbol{\mu}}{\boldsymbol{\mu}^{1}+\boldsymbol{\mu}}\right) \frac{\mathbf{x}}{\mathbf{b}}-\left(\frac{\mathbf{x}}{\mathbf{b}}\right)^{2}\right]
\end{aligned}
$$

\section{RESULTS}

\subsection{Pseudo mass transfer coefficient model for Extraction of Phenol:}

Using the pseudo mass transfer approach extraction ratio vs velocity flow for different $\mathrm{k}_{\mathrm{m}}$ are plotted. On reducing the $\mathrm{k}_{\mathrm{m}}$ values, it is found that the experimental data matches with the calculated data at $\mathrm{k}_{\mathrm{m}}=0.001$. 


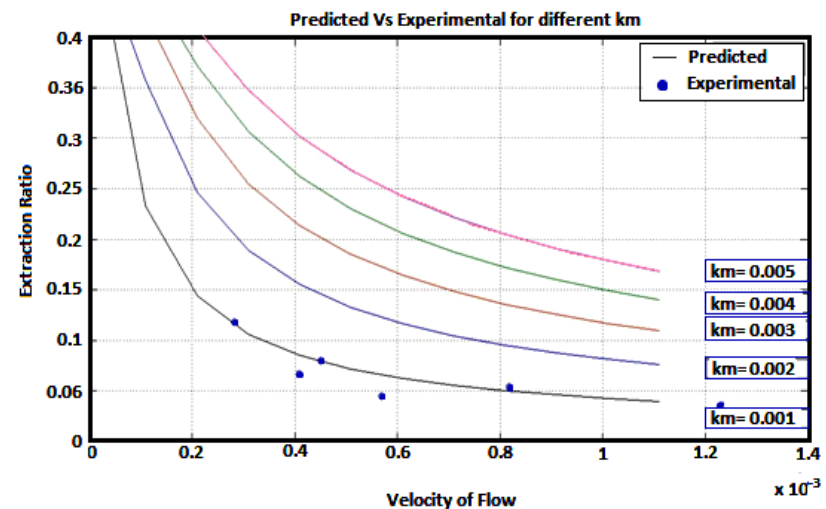

Fig 2: Predicted Vs experimental extraction ratio for different Km

\subsection{Plug velocity profile}

Assuming that both the fluids have same velocity $\mathrm{u}_{\mathrm{d}}=$ $\mathrm{u}_{\mathrm{w}}=0.0003$, extraction ratio is found out by calculating the concentration at different grid points.

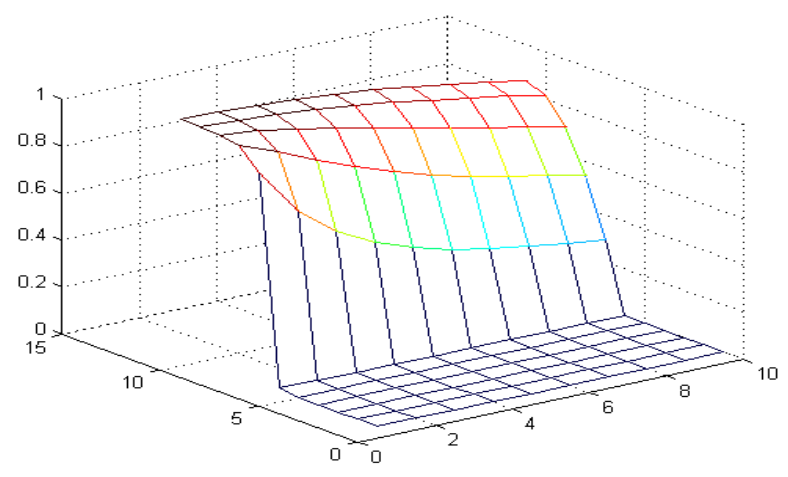

Fig 3 Concentration grids

The liquid is flowing from 0 to 10 in $\mathrm{x}$ direction. Upper (red) portion represents the dodecane concentration and the lower part gives the concentration of water. Sudden jump in the dodecane concentration shows the discontinuity arises in that area. Let the number of grids taken in $\mathrm{x}$ direction be 10 and that in $\mathrm{y}$ direction be 11 .

\section{Table 1: Concentration distribution for pseudo mass} transfer approach:

$\begin{array}{llllllllll}0.9995 & 0.9975 & 0.9921 & 0.9825 & 0.9686 & 0.9509 & 0.9300 & 0.9065 & 0.6821 & 0.8491 \\ 0.9990 & 0.9945 & 0.9848 & 0.9700 & 0.9508 & 0.9283 & 0.9035 & 0.8771 & 0.8506 & 0.8160 \\ 0.9960 & 0.9791 & 0.9520 & 0.9193 & 0.8842 & 0.8490 & 0.8146 & 0.7815 & 0.7507 & 0.7138 \\ 0.9822 & 0.9189 & 0.8500 & 0.7861 & 0.7301 & 0.6817 & 0.6400 & 0.6037 & 0.5724 & 0.5379 \\ 0.8946 & 0.6992 & 0.5767 & 0.4952 & 0.4378 & 0.3953 & 0.3625 & 0.3363 & 0.3150 & 0.2929 \\ 0.0118 & 0.0092 & 0.0076 & 0.0065 & 0.0057 & 0.0052 & 0.0047 & 0.0044 & 0.0041 & 0.0038 \\ 0.0004 & 0.0017 & 0.0024 & 0.0028 & 0.0030 & 0.0031 & 0.0031 & 0.0031 & 0.0030 & 0.0029 \\ 0.0000 & 0.0002 & 0.0005 & 0.0008 & 0.0011 & 0.0013 & 0.0014 & 0.0015 & 0.0016 & 0.0017 \\ 0.0000 & 0.0000 & 0.0001 & 0.0002 & 0.0003 & 0.0004 & 0.0005 & 0.0006 & 0.0007 & 0.0009 \\ 0.0000 & 0.0000 & 0.0000 & 0.0000 & 0.0001 & 0.0001 & 0.0001 & 0.0002 & 0.0002 & 0.0004 \\ 0.0000 & 0.0000 & 0.0000 & 0.0000 & 0.0000 & 0.0000 & 0.0001 & 0.0001 & 0.0001 & 0.0003\end{array}$

Calculated extraction ratio $=.28$

Experimental extraction ratio $=.118$

\subsection{Results for two dimensional mass transfer approach}

Velocity profile obtained by solving the momentum equation is inserted into the convection mass transfer equation to obtain the new concentration distribution.

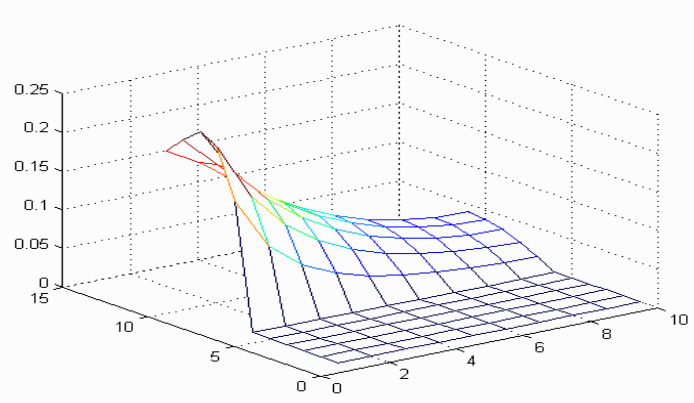

Fig 4: Concentration distribution for 2-D mass transfer approach.

Here the liquid is flowing from 0 to 10 in $\mathrm{x}$ direction. Upper (red) portion represents the dodecane concentration and the lower part gives the concentration of water in the figure 4 .

The number of grids taken in $\mathrm{x}$ direction is 10 and that in $\mathrm{y}$ direction is 11 .

$\begin{array}{llllllllll}0.1975 & 0.1746 & 0.1431 & 0.1136 & 0.0894 & 0.0705 & 0.0567 & 0.0472 & 0.0418 & 0.0399 \\ 0.2202 & 0.1790 & 0.1419 & 0.1111 & 0.0866 & 0.0680 & 0.0545 & 0.0453 & 0.0399 & 0.0381 \\ 0.2378 & 0.1771 & 0.1329 & 0.1005 & 0.0768 & 0.0596 & 0.0474 & 0.0391 & 0.0343 & 0.0327 \\ 0.2258 & 0.1538 & 0.1083 & 0.0787 & 0.0586 & 0.0448 & 0.0352 & 0.0289 & 0.0252 & 0.0240 \\ 0.1628 & 0.0969 & 0.0634 & 0.0442 & 0.0322 & 0.0243 & 0.0190 & 0.0155 & 0.0135 & 0.0128 \\ 0.0021 & 0.0013 & 0.0008 & 0.0006 & 0.0004 & 0.0003 & 0.0002 & 0.0002 & 0.0002 & 0.0002 \\ 0.0011 & 0.0010 & 0.0008 & 0.0006 & 0.0005 & 0.0004 & 0.0003 & 0.0003 & 0.0003 & 0.0003 \\ 0.0007 & 0.0008 & 0.0007 & 0.0006 & 0.0005 & 0.0005 & 0.0004 & 0.0004 & 0.0003 & 0.0003 \\ 0.0006 & 0.0007 & 0.0006 & 0.0006 & 0.0005 & 0.0005 & 0.0004 & 0.0004 & 0.0004 & 0.0004 \\ 0.0006 & 0.0006 & 0.0006 & 0.0006 & 0.0005 & 0.0005 & 0.0004 & 0.0004 & 0.0004 & 0.0004 \\ 0.0006 & 0.0006 & 0.0006 & 0.0006 & 0.0005 & 0.0005 & 0.0004 & 0.0004 & 0.0004 & 0.0004\end{array}$

Table 2: Concentration distribution for 2-D mass transfer approach

Extraction ratio calculated $=0.136$;

Extraction ratio experimental $=0.118$;

Therefore, the calculated value for two dimensional mass transfer approaches shows more matching than that of pseudo mass transfer approach with the experimental data. Hence better accuracy is expected with the incorporation of velocity profile into the convection diffusion equation. 


\subsection{Comparison of data:}

Table 3: Comparison of predictions with experimental

\begin{tabular}{|c|c|c|c|}
\hline Velocity & Experimental & $\begin{array}{c}\text { Pseudo Mass } \\
\text { Transfer } \\
\text { Model }\end{array}$ & $\begin{array}{c}\text { Two } \\
\text { Dimensional } \\
\text { Unsteady } \\
\text { diffusion } \\
\text { model }\end{array}$ \\
\hline 0.00028 & 0.118 & 0.1534 & 0.136 \\
\hline 0.0004 & 0.0668 & 0.0848 & 0.082 \\
\hline 0.00045 & 0.0795 & 0.077 & 0.078 \\
\hline 0.00057 & 0.0445 & 0.0622 & 0.0604 \\
\hline 0.000818 & 0.0541 & 0.0502 & 0.0532 \\
\hline 0.00123 & 0.035 & 0.0382 & 0.0343 \\
\hline
\end{tabular}

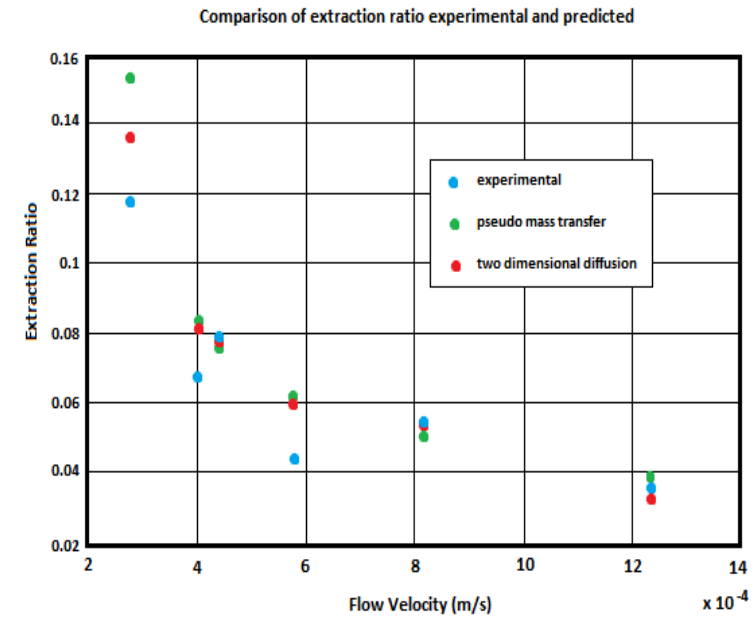

Fig 5: Comparison of the experimental data with model predictions.

\section{CONCLUSION}

The work done here concludes that, (1) Assumption of a plug velocity profile is insufficient and leads to large variation form experimental values. (2) Model involving the Pseudo mass transfer coefficient is simpler and demands less computational power. (3) Variation in predictions of both the models with flow velocities follow similar pattern. However, the predictions of the two dimensional diffusion model are closer to the experimental data but, at the expense of a higher computational requirement.

\section{REFERENCES}

[1] Anand Srinivasan, José L. López-Ribot, Anand K. Ramasubramanian: Microfluidic Applications in Vascular Bioengineering- IGI Global

[2] Kevin D. Nagy, Kla vs F. Jensen, 2011. Catalytic processes in small scale flow reactors: Status and opportunitiesChemistry Today.

[3] Stepan Spatenka, Vlastimil Fila, Bohumil Bernauer, Josef Fulem, Gabriele Germani, Yves Schuurman: Modelling and simulation of microchannel catalytic WGS reactor for an automotive fuel processor.

[4] Khamir Mehta, Jennifer J. Linderman: Model-Based Analysis and Design of a Microchannel Reactor for Tissue Engineering

[5] Matthew W. Losey, Rebecca J. Jackman, Samara L. Firebaugh, Martin A. Schmidt, Klavs F. Jensen: Design and Fabrication of Microfluidic Devices for Multiphase Mixing and Reaction

[6] Lennart Bitsch: Blood Flow in Microchannels

[7] George M. Whitesides: The origins and the future of microfluidics .

[8] Afzal Husain and Kwang-Yong Kim, 2008. Shape Optimization of Micro-Channel Heat Sink for MicroElectronic Cooling- IEEE Transactions On Components And Packaging Technologies

[9]. Dittrich, P. S. \& Manz, A. 2006. Lab-on-a-chip: microfluidics in drug discovery. Nature Rev. Drug Discov.

[10].Pihl, J., Karlsson, M. \& Chiu, D. T. 2005. Microfluidic technologies in drug discovery. Drug Discov. Today.

[11].Sia, S. K. \& Whitesides, G. M. 2003. Microfluidic devices fabricated in poly (dimethylsiloxane) for biological studies. Electrophoresis.

[12].Wheeler, A. R. et al. 2003. Microfluidic device for singlecell analysis. Anal. Chem.

[13].Werdich, A. A. et al. 2004. A microfluidic device to confine a single cardiac myocyte in a subnanoliter volume on planar microelectrodes for extracellular potential recordings. Lab Chip.

[14].Y Okubo, T Maki, N Aoki, 2008. Liquid-liquid extraction for efficient synthesis and separation by utilizing micro spaces- Chemical Engineering Science.

[15].R. Byron Bird. 2001. Transport Phenomena, Second Edition.

[16].Smirnova, A., Shimura, K., Hibara, A., Proskurnin, M.A., Kitamori, T., 2007. Application of a micro multiphase laminar flow on a microchip for extraction and determination of derivatized carbanate pesticede. Analytical Sciences 23, 103-107.

[17].Shaw, J., Nudd, R., Naik, B., Turner, C., Rudge, D., Benson, M., Garman, A., 2000. Liquid/ liquid extraction systems using micro-contactor arrays. Micro Total Analysis System, 371-374. 